\title{
Efficacy of Epalrestat and Pregabalin in Patients with Diabetic Peripheral Neuropathy
}

\author{
Ramya Shruthi $\mathrm{K}^{1}$, Gopala Rao $\mathbf{P}^{1}$, Anushma $\mathbf{R}^{1}$, Bandaru Siva Subrahmanyam ${ }^{2}$, Eggadi Venkateshwarlu ${ }^{1}$ and \\ Bandaru SSB ${ }^{1 *}$ \\ ${ }^{1}$ Department of Clinical Pharmacy, India \\ ${ }^{2}$ Sri Bhadrakali Diabetic Clinic, India
}

*Corresponding author: Bandaru SSB, Department of Clinical Pharmacy, Vaagdevi College of Pharmacy, Warangal, India; Email: sharavanabhava6@gmail.com

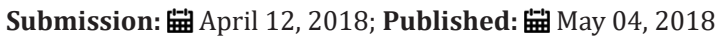

\begin{abstract}
Diabetic Peripheral neuropathy [DPN] is a major long term problem allied with diabetes that can cause serious disability and also death. It is caused by damage to any nerve in the peripheral nervous system. Fifty to seventy five percent of all ulcerations and non trauma amputations are a consequence of diabetic neuropathy. Epalrestat and Pregabalin are widely used to overcome neuronal damage. This study was designed to evaluate the efficacy of these two drug regimens.
\end{abstract}

Material and methods: Patients included in this study were experiencing pain because of diabetic neuropathy for at least 6 months to 2 years.

Results: From 256 subjects with diabetic neuropathy included in the study, 229 patients concluded final analysis. 27 patients dropped from the study (17and 10 patients from pregabalin and epalrestat respectively). Mean pain score was reduced from $5.08 \pm 0.82$ (severe pain) at first visit to $3.43 \pm 0.93$ (moderate pain) in the epalrestat group, from $6.42 \pm 1.01$ (severe pain) at first visit to $2.57 \pm 0.59$ (mild pain) in the pregabalin group.

Conclusion: We conclude that pregabalin was significantly more effective than epalrestat in controlling pain in DPN patients.

Keywords: Diabetic peripheral neuropathy; Epalrestat and pregabalin

\section{Background and Aims}

Diabetic neuropathy (DPN) encompasses a wide, heterogeneous group of clinical and subclinical syndromes [1]. It is a major long term problem allied with diabetes that can cause serious disability and also death [2]. 50 to $75 \%$ of all ulceration and non-traumatic amputations are a consequence of diabetic neuropathy, and cause more hospitalizations than all other diabetic complications [3]. DN affects the nervous system and causes extensive damage. Neurologic complications are not reserved for specific type of diabetes but occur equally in type 1 and type 2 [4]. Diabetic peripheral neuropathy (DPN) is often painful and debilitating condition that is caused by damage to any nerve in the peripheral nervous system.

It is a family of nerve disorders that are directly caused by diabetic complications [5]. Poor diabetic control, obesity, high blood pressure, high cholesterol and triglycerides are risk factors for developing neuropathy [6]. It affects somatic and autonomous nervous systems and is different from peripheral arterial disease which affects the blood vessels rather than the nerves and vasa nervorum [7]. Many physicians misinterpret symptoms related to neuropathy in diabetic patients. Treatment is directed towards preventing neuropathy progression, reducing symptoms and implementing measures to prevent complications of insensate extremities [8].

The aim of this study was to analyze the effect on neuropathic pain of two widely used drugs: epalrestat and Pregabalin.

\section{Material and Methods}

This was a prospective observational study carried out in Sri Bhadrakali Diabetic Clinic, Naimnagar, Hanamkonda. Institutional Human Ethics committee endorsement was seeked and obtained before conduct of the trial (MGM/VCOP/PHARMD/V/007/2017). Selection of subjects was done according to the following inclusionexclusion criteria:

\section{Inclusion criteria}

Males and females 18-75 years age; Diabetes mellitus (Type1 or Type2); Experiencing pain due to diabetic neuropathy for at least 6 months to 2 years; Neuropathic pain must begin in the feet with 
relatively symmetrical onset; Patients who had Douler neuropathic pain questionnaire [DN4] Score 4 or more and patients who had a Vibration Perception Threshold (VPT) score of above 20.

\section{Exclusion criteria}

Suffering from ischemic pain and other types of pain unrelated to diabetic neuropathy; History of substance abuse or dependence within the past year excluding nicotine and caffeine. Pregnancy or breast-feeding; concomitant medical exclusion included, chronic use of anti-depressants, analgesics, antimanics, anti-migraines, antipsychotics, benzodiazepines, capsaicin chloral hydrate and guanethidine. Psychological conditions that might compromise participation in the study; Any clinically significant neurological disorders, significant unstable medical or psychiatric conditions that would interfere with the patient's ability to participate in the study.

Written and oral informed consent forms were obtained and evaluated before any study procedures. The patient disposition is given in Figure 1. Thus, a total of 256 subjects with diabetic neuropathy (DPN) were included in the study. They were randomized in two study groups, to receive Pregabalin (Group 1), and epalrestat (Group 2). Of these, 27 patients dropped from the study (17and 10 patients from the Pregabalin and epalrestat groups respectively).

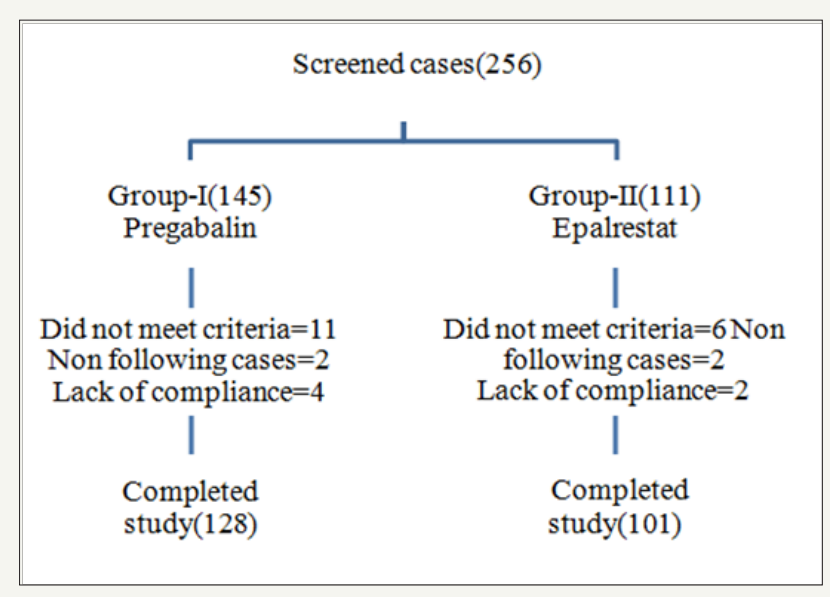

Figure 1: Distribution of patients according to DPN guidelines.

Patient's medical history and demographic details were documented at screening visit. Before starting the therapy, first parameters were evaluated from patient's records. Pain scores were confirmed by DN4 Questionnaire and VPT was evaluated with a biothesiometer. During the course of the trial, progress of patients was tracked using the Douler's neuropathic pain questionnaire (DN4) by assessing burning, itching, and numbness, loss of heat and cold sensations, electric shocks, tingling and brushing symptoms. Clinic and laboratory collected data included weight, systolic and diastolic blood pressure (BP), glycated hemoglobin (HbA1C), Fasting Blood Glucose (FBG), Post Prandial blood Glucose (PPG), serum creatinine, serum electrolytes, microalbuminuria, hemoglobin and lipid profiles.

Vibration Perception Threshold (VPT) and intensity of pain were witnessed at begining and end of the study. Comparison of pain score was done with DN4 Questionarrie [9].

\section{Measurements of treatment efficacy}

The primary efficacy measure for this study was the reduction of pain scores. Subjective pain was assessed by DN4 Questionnaire and rated by patients. Patients ratings were tabulated for calculation of mean scores at baseline and endpoint. The DN4 severity subscores included, if score is $4 / 10$ not a neuropathic pain, if score is $>4 / 10$ indicate neuropathic pain.
The secondary efficacy measures collected were vibration perception threshold (VPT) by using a biothesiometer. The VPT by using biothesiometer has been used to identify peripheral sensory neuropathy and subjects at risk of foot ulcerations. The biothesiometer is a rapid, portable, and sensitive method of assessing VPT and has been used to identify subclinical neuropathy and to monitor the progress of the disease. It allows increasing the vibrating strength and measuring the threshold of vibration perception by gradually increasing the vibrations. Interpretation of the results is made using a biothesiometric score as detailed in Table 1. Patients with a VPT greater than 25 were considered to have a significant neuropathy.

Table 1: Biothesiometer score interpretation.

\begin{tabular}{|c|c|}
\hline Severity & VPT \\
\hline Normal & Up to15 volts \\
\hline Grade 1 & $16-25$ volts \\
\hline Grade- 2 & $>25$ volts \\
\hline
\end{tabular}

\section{Testing vibration sensation with the biothesiometric}

A probe is applied on the foot, usually on the big toe. The probe can be made to vibrate at increasing intensity by tuning a dial. The person being tested indicates as soon as he/she can feel the vibration and the reading on the dial at that point are recorded. 
The biothesiometer has a reading from $0-50$ volts. Data from the literature show that the risk of developing a neuropathic ulcer is much higher if a person has a biothesiometer reading greater than $30-40$ volts [9].

\section{Statistical analysis}

Data analysis was done using Graph Pad Prism software (version 5). Mean and Standard deviation (SD) were calculated for the normal distributed variables efficacy measures, laboratory measures and vital signs. Difference between quantitative variables was evaluated by using the Paired t test.

\section{Results}

Demographic characteristics of the study population are given in Table 2 .

Table 2: Patient demographic characteristics of the study population $(n=229)$.

\begin{tabular}{|c|c|}
\hline Characteristics & Values (Mean \pm SD) \\
\hline Age (years) & $54 \pm 10$ \\
\hline Male n (\%) & $58(40 \%)$ \\
\hline Female n (\%) & $87(60 \%)$ \\
\hline Rural n (\%) & $80(55 \%)$ \\
\hline Urban n (\%) & $65(45 \%)$ \\
\hline Weight (kg) & $62.1 \pm 11.3$ \\
\hline BMI $\left(\mathrm{kg} / \mathrm{m}^{2}\right)$ & $24.4 \pm 3.22$ \\
\hline Duration of diabetes (years) & $8.02 \pm 4.23$ \\
\hline
\end{tabular}

\section{VPT assessed with the biothesiometer in the right leg}

At Visit 1 the mean VPT score of Epalrestat [E] was 18.26 \pm 3.23 and decreased at Visit 2 to $16.08 \pm 3.29$ and in the Pregabalin group $[\mathrm{P}]$, the mean VPT score also decreased from Visit $1(16.99 \pm 3.4)$ to Visit 2 (12.7 \pm 2.94$)$ as detailed in Figure 2.

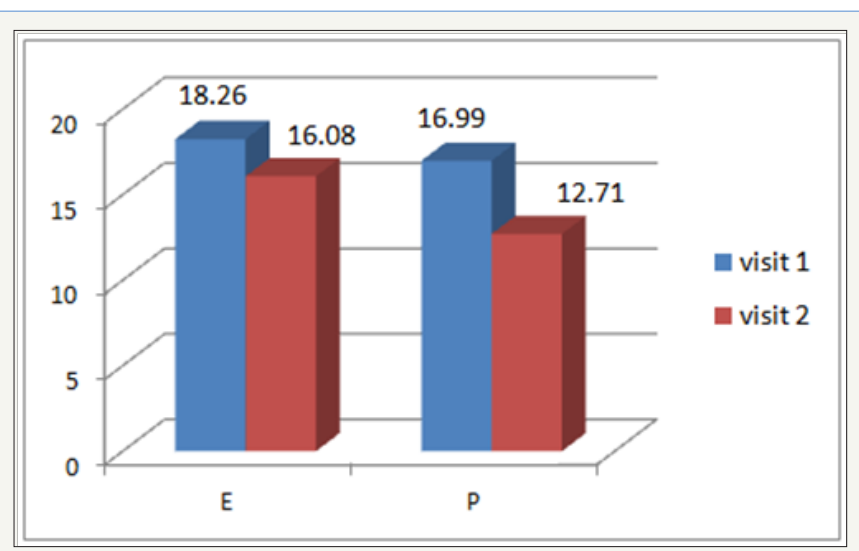

Figure 2: Right leg mean VPT score of the 2 drugs, at Visit 1 and Visit 2.

Comparing the improvement of the VPT score in the 2 study groups we observed that the reduction of the VPT score was significantly higher with Pregabalin $(4.28 \pm 0.32)$ when compared with epalrestat $(2.18 \pm 0.06)$ and percentage reduction was (25.19\%) (11.93\%) for Pregabalin and Epalrestat respectively.

\section{VPT assessed with the biothesiometer in the left leg}

At Visit 1, the mean VPT score of Epalrestst [E] was 18.69 \pm 3.17 and decreased at Visit 2 to $16.93 \pm 3.61$ and in the Pregabalin group $[\mathrm{P}]$, the mean VPT score also decreased from Visit $1(17.55 \pm 3.47)$ to Visit $2(13.18 \pm 2.93)$ as detailed in Figure 3.

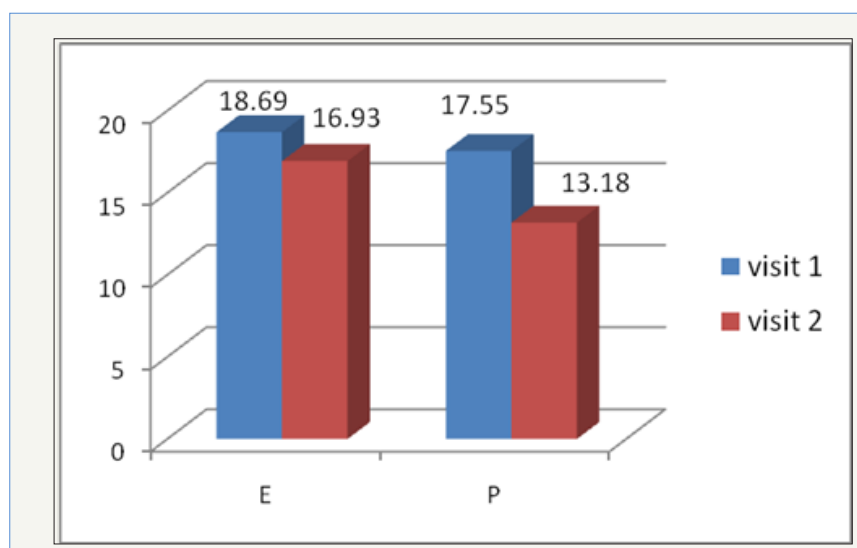

Figure 3: Left leg mean VPT Score of the two study groups, at Visit 1 and Visit 2.

Comparing the improvement of the VPT score in the 2 study groups we observed that the reduction of the VPT score was higher in Pregabalin $(4.37 \pm 0.53)$ when compared with epalrestat $(1.76 \pm 0.44)$ and percentage reduction was $(24.9 \%)(9.41 \%)$ for Pregabalin and Epalrestat respectively.

\section{Effect of treatment on the pain intensity score}

In the first group [E], pain intensity score decreased from $5.08 \pm 0.82$ (severe pain) at Visit1 to $3.43 \pm 0.93$ (moderate pain) at Visit 2 and from $6.42 \pm 1.01$ (severe pain) at baseline to $2.57 \pm 0.59$ (mild pain) at the end of the treatment for the second group [P] respectively, as detailed in Figure 4. Comparing the DN4 Questonnaire improvement for the two study groups, the mean difference of pain intensity was higher in the $[\mathrm{P}]$ group $(3.85 \pm 0.42)$ when compared with [E] group $(1.65 \pm 0.11)$.

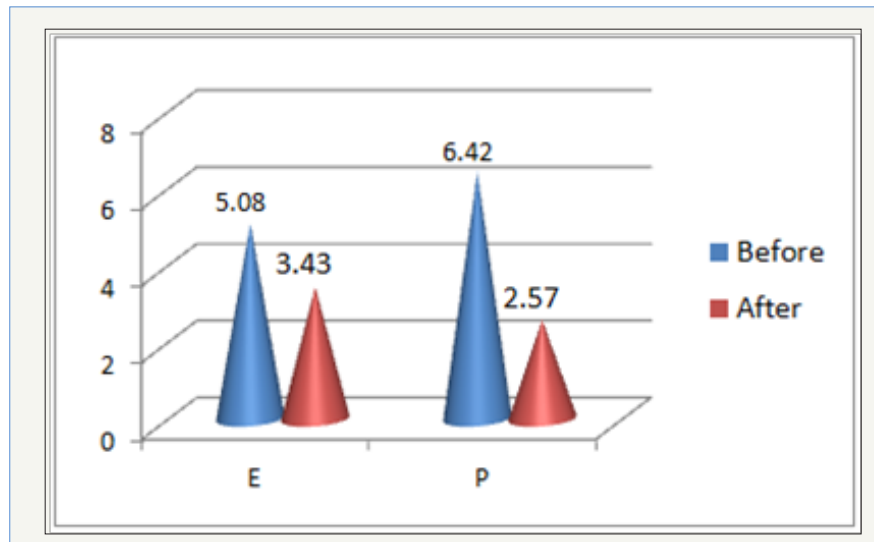

Figure 4: Pain intensity score in the three study groups at Visit 1 and Visit 2. 


\section{Discussion}

Poor glycemic control is a key factor for the development of diabetic neuropathy. Specific treatment of neuropathic pain leads to an improvement in the quality of life of these patients. This research was carried out to assess the efficacy and safety of two available treatment regimens in patients with diabetic neuropathy. Loss of sensation, numbness, weakness, pain, loss of thermal sensitivity, burning sensation and muscle cramps have been evaluated in this study, all these being the most common diabetic neuropathy complaints.

A great deal of evidence suggests that Treatment with Anticonvulsants resulted in lower pain intensity and greater pain relief. Various other studies have shown that Pregabalin may be useful in treating pain associated with diabetic neuropathy. Pregabalin have a higher efficacy for interacting with noradrenergic and serotonergic pathways originating from brain stem which reduce neuropathic pain transmission. It is effective to treat pain in animal models. The ability of Pregabalin to exert its effects on both these pathways may explain its effectiveness in this clinical trial. Pregabalin was more safe and effective in decreasing pain associated with DPN, and also improved mood, sleep disturbance, and quality of life [10]. Epalrestat interferes potentially by preventing or diabetic neuropathy. Epalrestat helps in preventing degeneration of neurons by decreasing the sorbitol accumulation and reducing the oxidative stress while methylcobalamine heals the injury [11].

The risk factors for DPN- Hypertension, high cholesterol, increased age, BMI, triglycerides, HDL, etc. Evaluation of these parameters become mandatory.

This research revealed that all the two drugs had a positive effect on pain intensity scores of patients with diabetic neuropathy. No significant difference in laboratory parameters was observed. For all patients' good glycemic control, lipid management, maintaining healthy life style and monitoring of all parameters of diabetes management as advised by the physicians are of paramount importance. The fact that the two drugs tested in this study did not change the laboratory parameters during the study period may indicate that these drugs are safe in using DPN patients.

\section{Conclusion}

We conclude that pregabalin was significantly more effective than Epalrestat in controlling pain in DPN patients.

\section{References}

1. Dub JJ, Campbell RK, Setter SM, Rasmussen KA (2004) Diabetic neuropathy: an intensive review. Am J Health Syst Pharm 61(2): 160173.

2. Hare JA, Abuaisha F, Geoghegan M (1994) Prevalence and forms of neuropathic morbidity in 800 diabetics. J Med Sci 163(3): 132-135.

3. Bhadada SK, Sahay RK, Jyotsna VP, Agrawal JK (2001) Diabetic neuropathy: current concepts. Journal of Indian Academy of Clinical Medicine 2: 305-318.

4. Boulton AJ, Malik RA, Arezzo JC, Sosenko JM (2004) Diabetic somatic neuropathies. Diabetes Care 27(6): 1458-1486.

5. Harati Y (1987) Diabetic peripheral neuropathies. Annals Internal Medicine 107: 546-559.

6. Tesfaye S, Chaturvedi N, Eaton SE, Ward JD, Manes C, et al. (2005) Vascular risk factors and diabetic neuropathy. NEJM 352: 341-350.

7. Cobb W, Lee L (2012) Peripheral diabetic neuropathy cost effectiveness should be an important consideration. Advance NPs PAs 3: 18-21.

8. Tesfaye S, Boulton AJ, Dyck PJ, Horowitz M, Freeman R, et al. (2010) Diabetic neuropathies: update on definitions, diagnostic criteria, estimation of severity, and treatments. Diabetes Care 33(10): 22852293.

9. Yagihashi S, Mizukami H, Sugimoto K (2011) Mechanism of diabetic neuropathy: Where are we now and where to go? J Diabetes Investig 2(1): 18-32.

10. Rosenstock J (2004) pregabalin for the treatment of painful diabetic peripheral neuropathy: a double blind, placebo-controlled trial. Pain 110(3): 628-638.

11. Yagihashi S, Yamagishi S, Wada R (2007) Pathology and pathogenetic mechanisms of diabetic neuropathy: correlation with clinical signs and symptoms. Diabetes Res Clin Pract 77(Supply 1): 184-189.

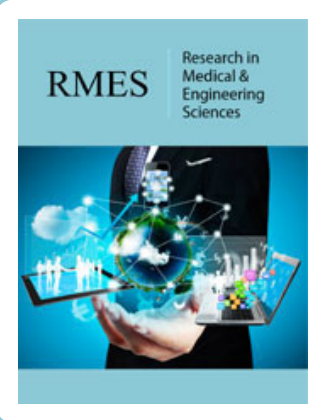

\section{Research in Medical \& Engineering Sciences}

\section{Benefits of Publishing with us}

- High-level peer review and editorial services

- Freely accessible online immediately upon publication

- Authors retain the copyright to their work

- Licensing it under a Creative Commons license

- Visibility through different online platforms 